\title{
Contexto de trabalho e Prazer-sofrimento na Atenção Primária à Saúde
}

\author{
Work context and Pleasure-suffering in Primary Health Care \\ Contexto de trabajo y Placer-sufrimiento en la Atención Primaria de Salud
}

Recebido: 25/01/2021 | Revisado: 26/01/2021 | Aceito: 28/01/2021 | Publicado: 06/02/2021

\author{
Graziele de Lima Dalmolin \\ ORCID: https://orcid.org/0000-0003-0985-5788 \\ Universidade Federal de Santa Maria, Brasil \\ E-mail: grazi.dalmolin@gmail.com. \\ Taís Carpes Lanes \\ ORCID: https://orcid.org/0000-0001-9337-7875 \\ Universidade Federal de Santa Maria, Brasil \\ E-mail: taislanes_rock@hotmail.com \\ Roosi Eloiza Bolzan Zanon \\ ORCID: https://orcid.org/0000-0002-4033-5361 \\ Universidade Federal de Santa Maria, Brasil \\ E-mail: roosi.zanon@gmail.com \\ Thaís Costa Schutz \\ ORCID: https://orcid.org/0000-0003-4341-0395 \\ Universidade Federal de Santa Maria, Brasil \\ E-mail: thais.schutz@hotmail.com \\ Katiane Sefrin Speroni \\ ORCID: https://orcid.org/0000-0001-6815-1559 \\ Universidade Federal de Santa Maria, Brasil \\ E-mail: katiane.speroni@gmail.com
}

\begin{abstract}
Resumo
Objetivo: Avaliar o risco de adoecimento relacionado ao contexto de trabalho e ao prazer e sofrimento no trabalho de enfermeiros e médicos da Atenção Primária à Saúde. Métodos: Estudo transversal realizado com 76 profissionais médicos e enfermeiros de 34 unidades da Atenção Primária à Saúde de um município do Rio Grande do Sul, Brasil. A coleta de dados aconteceu de março a agosto de 2015, por meio da Escala de Avaliação do Contexto de Trabalho e da Escala de Indicadores de Prazer e Sofrimento no Trabalho. Para análise dos dados, utilizou-se estatística descritiva e analítica. Resultados: Os enfermeiros e médicos avaliaram o contexto de trabalho como crítico em dois fatores: "Organização do trabalho" e "Condições de trabalho". O fator "Relações socioprofissionais" foi avaliado como satisfatório, somente pelos médicos. No que diz respeito à vivência de prazer e sofrimento, os fatores "Realização profissional" e "Esgotamento profissional" foram avaliados como críticos e "Liberdade de expressão" foi considerado satisfatório, para ambas categorias. O fator "Falta de reconhecimento" foi satisfatório somente sob a percepção dos médicos. Entre os fatores das duas escalas, ocorreu correlação forte e significativa entre o fator "Organização do Trabalho" e "Esgotamento profissional" e entre "Relações socioprofissionais" e "Falta de reconhecimento". Conclusão: O contexto de trabalho reflete risco moderado para o adoecimento destes profissionais, em que enfermeiros sinalizaram, com avaliações mais críticas que os médicos, para os fatores de cada uma das escalas, exceto para o fator "Liberdade de expressão".
\end{abstract}

Palavras-chave: Condições de trabalho; Saúde do trabalhador; Atenção primária à saúde; Enfermagem.

\begin{abstract}
Objective: To assess the risk of illness related to the work context and the pleasure-suffering of nurses and doctors at Primary Health Care. Methods: This transversal study was conducted with 76 doctors and Nursing professionals of 34 Primary Care units in a city of Rio Grande do Sul, Brazil. Data was collected between march to august 2015 by the Scale for Assessment of the Context of Work and the Scale of Indicators of Pleasure-Suffering at Work. For the analysis of data descripted and analytical statistics were used. Results: Nursing professionals and doctors assessed the context of work as critical by two factors: "Work organization" and "Work conditions". The "socio-professional relationships" factor was assessed as satisfactory only by the doctors. About the pleasure-suffering daily routine, "professional realization" and "professional exhaustion" factors were assessed as critical and "freedom of speech" was considered satisfactory for both of categories. The "lack of recognition" factor was considered satisfactory only by the perception of doctors. Between the factors of both scales, a strong and significant correlation occurred in "Work organization" and "Work exhaustion" and between "socio-professional relationships" and "lack of recognition. Conclusion: The work context reflects moderate risk for illness of these professionals in which nurses signaled, with more critical assessments than doctors, for the factors of each of the scales, except for the "freedom of speech" factor. Keywords: Working conditions; Occupational health; Primary health care; Nursing.
\end{abstract}




\section{Resumen}

Objetivo: evaluar el riesgo de enfermedad relacionado con el contexto de trabajo y al placer y sufrimiento en el trabajo de enfermeros y médicos de la Atención Primaria de Salud. Métodos: Estudio transversal realizado con 76 profesionales médicos y enfermeros de 34 unidades de la Atención Primaria de Salud en un municipio de Rio Grande do Sul, Brasil. La recolección de datos se realizó de marzo a agosto de 2015, utilizando la Escala de Evaluación del Contexto de Trabajo y la Escala de Indicadores de Placer y Sufrimiento en el Trabajo. Para el análisis de datos, se utilizaron estadística descriptiva y analítica. Resultados: Los enfermeras y médicos evaluaron el contexto de trabajo como crítico en dos factores: "Organización del trabajo" y "Condiciones de trabajo". El factor "Relaciones socioprofesionales" fue evaluado como satisfactorio, solo por médicos. Con respecto a la experiencia de placer y sufrimiento, los factores "Realización profesional" y "Agotamiento profesional" fueron evaluados como críticos y "Libertad de expresión" fue considerada satisfactoria, para ambas categorías. El factor "Falta de reconocimiento" fue satisfactorio solamente por la percepción de los médicos. Entre los factores de las dos escalas, hubo correlación fuerte y significativa entre el factor "Organización del trabajo" y "Agotamiento profesional" y entre "Relaciones socioprofesionales" y "Falta de reconocimiento". Conclusión: El contexto de trabajo refleja un riesgo moderado para enfermedad de estos profesionales, en que enfermeros señalaron, con evaluaciones más críticas que los médicos, para los factores de cada una de las escalas, excepto para el factor "Libertad de expresión".

Palabras clave: Condiciones de trabajo; Salud laboral; Atención primaria de salud; Enfermería.

\section{Introdução}

A Atenção Primária à Saúde (APS) engloba um conjunto de ações que envolvem a promoção, prevenção, proteção, diagnóstico, tratamento e reabilitação dos usuários, incluindo cuidados paliativos e vigilância em saúde. Essas ações devem ser desenvolvidas por equipe multiprofissional de um determinado território, com aplicação de práticas de cuidado integrado e gestão qualificada (Brasil, 2017).

Considerada a porta de entrada do usuário no Sistema Único de Saúde (SUS), a APS possui uma ampla lista de atribuições aos seus profissionais. Essas atribuições são baseadas em normativas específicas do Ministério da Saúde (MS), incluindo "escopo de práticas, protocolos, diretrizes clínicas e terapêuticas", além de outras normativas da gestão municipal, estadual e federal (Brasil, 2017).

O trabalhador da APS tem o desafio de operacionalizar os princípios e diretrizes do SUS. Esta operacionalização está condicionada a complexos fatores, que exigem esforço político-institucional quanto ao financiamento, à gestão e à formação dos profissionais. Isso requer participação social nas ações de planejamento e organização, para atender às necessidades da população, a fim de superar processos de trabalho regidos pelo modelo biomédico, e garantir os cuidados integrais de saúde (Brasil, 2017; Miranda, Rewa, Leonello, \& Oliveira, 2018).

A participação deste trabalhador na organização e planejamento de ações dentro desses serviços, torna-o exposto a riscos ocupacionais, tais como: químicos, físicos, ergonômicos e biológicos, bem como os advindos da situação social e local, onde se insere a comunidade de atuação do profissional (Wagner, et al., 2019). As pesquisas sobre a relação saúde-trabalho na APS têm focado a exposição à violência, as cargas laborais, o cotidiano de trabalho e seus desafios (Dalmolin, et al., 2020; Sousa, Gonçalves, Silva, Soares, Nogueira, \& Zeitoune, 2018; Oliveira, Costa, Fernandes, Gouveia \& Rocha, 2018). Esses aspectos, juntamente com a forma de organização do trabalho, podem contribuir para o adoecimento e sofrimento do trabalhador.

Nesse sentido, o referencial da Psicodinâmica do Trabalho acena com um método de investigação, baseado no pressuposto de que o trabalho ocupa um lugar central na construção da identidade, das formas de sociabilidade e da autoestima, bem como na determinação do sofrimento psíquico (Dejours, 2004). Essa abordagem, apresenta-se como relevante referencial teórico no campo da saúde mental e no contexto de trabalho, pois além de uma disciplina clínica, ela é também uma teoria centrada na causa de sofrimento e prazer do trabalhador (Dejours, 2013).

Diante desses aspectos, ao se considerar que o trabalho pode ser fonte de sofrimento quanto de prazer, mediados pelo contexto de trabalho, destaca-se que a atuação de médicos e enfermeiros na APS envolve essas duas naturezas. As carências 
estruturais da APS dificultam as condições de trabalho (Schneider, Wehler \& Weigl, 2019), principalmente pela escassez ou falta de recursos, em que são apontados como aspectos negativos para qualidade de vida no trabalho dos profissionais de saúde (Schneider, Wehler \& Weigl, 2019).

A relevância desta investigação se acentua nas atuais circunstâncias, onde se projeta aumento das exigências ao trabalhador com a redução de equipes. Isso representa uma dificuldade na atenção integral, em que implica no aumento de riscos à saúde dos trabalhadores e redução da atenção à saúde dos usuários da APS. Assim, apresentou-se como objetivo avaliar o risco de adoecimento relacionado ao contexto de trabalho e ao prazer e sofrimento no trabalho de enfermeiros e médicos da Atenção Primária à Saúde.

\section{Metodologia}

Estudo quantitativo com delineamento transversal (Pereira, 2018), realizado em todas as unidades da APS, de um município do Estado do Rio Grande do Sul, Brasil. Neste município, a APS dispõe de 34 unidades de saúde, das quais 19 são Unidades Básicas de Saúde (UBS), 13 Estratégias de Saúde da Família (ESF) e duas unidades de saúde são definidas como mistas, devido ao processo de reorganização e reformulação das equipes da APS no município. As unidades mistas contam com duas equipes que atuam na assistência.

Considerou-se a população deste estudo, 124 profissionais enfermeiros e médicos atuantes na APS do município pesquisado. Para fins estatísticos, considerou-se uma amostra não probabilística por conveniência. Todos os enfermeiros e médicos que estavam nos seus locais de trabalho, durante coleta de dados, foram convidados a participar, sendo que amostra foi constituída por 76 participantes.

Adotou-se como critério de inclusão ser trabalhador da APS e estar atuando há pelo menos seis meses como enfermeiro ou médico no setor. Foram excluídos os trabalhadores que estavam em férias ou licença, de qualquer natureza, no período da coleta de dados.

Para coleta, utilizou-se um instrumento de caracterização sociodemográfica e laboral (sexo, idade, número de filhos, local de trabalho, profissão, tempo de formação, satisfação com salário, grau de satisfação com trabalho, turno de trabalho, vínculo empregatício, outro emprego e tempo médio de serviço) e as duas escalas que compõem o Inventário do Trabalho e Riscos de Adoecimento (ITRA): a Escala de Avaliação do Contexto de Trabalho (EACT) e a Escala de Indicadores de Prazer e Sofrimento no Trabalho (EIPST) (Mendes, Ferreira \& Cruz, 2007), as quais foram elaboradas e validadas no Brasil (Mendes, Ferreira \& Cruz, 2007).

A EACT avalia o contexto de trabalho, sendo composta por 31 itens divididos em três fatores: Organização do trabalho (questões um a 11); Condições de trabalho (questões 22 a 31) e Relações socioprofissionais (questões 12 a 16), pontuados por meio de uma escala do tipo Likert de cinco pontos, variando de $1=$ nunca, $2=$ raramente, $3=$ às vezes, $4=$ frequentemente a $5=$ sempre. A análise da EACT permite avaliar o risco de adoecimento em relação ao contexto de trabalho, conforme a média da pontuação: avaliação mais negativa, grave $(\geq 3,7)$, avaliação moderada, crítico $(2,3$ a 3,69) e avaliação mais positiva, satisfatório $(\leq 2,29)$ (Mendes, Ferreira \& Cruz, 2007).

A EIPST avalia a vivência dos indicadores de prazer e sofrimento do trabalhador, a qual é composta por 32 itens divididos em quatro fatores, dois que avaliam o prazer no trabalho: Realização profissional (questões nove a 17) e Liberdade de expressão (questões um a oito); e dois que avaliam o sofrimento: Esgotamento profissional (questões 18 a 24 ) e Falta de reconhecimento (questões 25 a 32). São pontuados por uma escala Likert de sete pontos, na qual: $0=$ nenhuma vez, $1=$ uma vez, 2 = duas vezes, 3 = três vezes, $4=$ quatro vezes, $5=$ cinco vezes e $6=$ seis vezes ou mais, correspondendo a quantas vezes o trabalhador vivenciou a situação no trabalho (Mendes, Ferreira \& Cruz, 2007). 
Para "Realização profissional" e "Liberdade de expressão" temos avaliação positiva ou satisfatória (média acima de 4,0), avaliação moderada ou crítica (média entre 2,1 e 3,9) e avaliação grave (média igual ou abaixo de 2,0). Os fatores "Esgotamento profissional" e "Falta de reconhecimento" apresentam avaliação negativa, grave (média acima de 4,0), moderada, crítico (média entre 2,1 e 3,9) e avaliação menos negativa, satisfatória (média igual ou abaixo de 2,0) (Mendes, Ferreira \& Cruz, 2007).

A coleta de dados aconteceu entre março a agosto de 2015, em turno diurno de segunda a sexta-feira, por coletadores previamente capacitados. Antes de iniciar a coleta de dados foi acordado com os coordenadores das unidades de saúde o agendamento das coletas, de modo que não prejudicasse o fluxo de trabalho.

Os enfermeiros e médicos de cada uma das unidades da APS foram convidados a participar do estudo, os quais receberam orientações referente aos objetivos da pesquisa. Os profissionais que concordaram em participar, assinaram o Termo de Consentimento Livre e Esclarecido, em duas vias, e responderam individualmente ao instrumento no local e horário de trabalho.

Os dados foram digitados no Software Microsoft Excel ${ }^{\circledR}$, com dupla digitação independente, verificação de erros e inconsistências e, posteriormente, exportados para o programa Predictive Analytics Software Statistic® (PASW da SPSS Inc., Chicago, USA) versão 17.0 para Windows. A verificação da confiabilidade das escalas foi testada por meio do alfa de cronbach, considerando valores satisfatórios acima de 0,70 (Hair, Black, Babin, Anderson \&Tatham, 2009). As variáveis contínuas foram descritas pelas médias e desvio padrão, e as categóricas foram descritas por frequências absolutas e relativas.

As pontuações médias da EACT e EIPST, e dos respectivos fatores, foram avaliadas separadamente e interpretadas considerando as médias de cada escala (Mendes, Ferreira \& Cruz, 2007). A correlação entre a EACT e a EIPST foi realizada por meio da correlação de Pearson, adotando-se nível de significância de $5 \%(\mathrm{p} \leq 0,05)$. A classificação da intensidade da correlação adotada foi: 0,1 a 0,29 correlação fraca; de 0,3 a 0,49 correlação moderada e acima de 0,5 é considerada correlação forte (Bisquerra, Sarriera \& Martínez, 2004).

Foram seguidos os preceitos éticos conforme a Resolução 466/12 (Brasil, 2013), do Conselho Nacional de Saúde/MS, com parecer favorável a realização pelo Comitê de Ética em Pesquisa (CEP) da Universidade Federal de Santa Maria, com Certificado de Apresentação para Apreciação Ética número 40264314.4.0000.5346, e número de parecer 932.842, em 12 de janeiro de 2015 .

\section{Resultados}

Participaram do estudo 76 profissionais, sendo 59,2\% (n=45) enfermeiros e 40,8\% (n=31) médicos. Na Tabela 1, são expostos os dados sociodemográficos e laborais dos participantes. 
Tabela 1. Dados sociodemográficos e laborais dos participantes $(n=76)$.

\begin{tabular}{|c|c|c|}
\hline \multirow[b]{2}{*}{ Variáveis } & \multirow{2}{*}{$\begin{array}{l}\text { Enfermeiros }(n=45) \\
n(\%)\end{array}$} & \multirow{2}{*}{$\begin{array}{l}\text { Médicos }(\mathrm{n}=31) \\
\mathrm{n}(\%)\end{array}$} \\
\hline & & \\
\hline \multicolumn{3}{|l|}{ Filhos } \\
\hline Sim & $29(64,4)$ & $18(58,1)$ \\
\hline Não & $16(35,6)$ & $13(41,9)$ \\
\hline \multicolumn{3}{|l|}{ Situação conjugal } \\
\hline Sem companheiro & $15(33,4)$ & $12(38,7)$ \\
\hline Com companheiro & $30(66,6)$ & $19(61,3)$ \\
\hline \multicolumn{3}{|c|}{ Possui outro emprego } \\
\hline $\operatorname{Sim}$ & $5(11,1)$ & $16(51,6)$ \\
\hline Não & $40(88,9)$ & $15(48,4)$ \\
\hline \multicolumn{3}{|l|}{ Escolaridade } \\
\hline Graduação & $4(8,9)$ & $16(51,6)$ \\
\hline Pós-graduação & $41(91,1)$ & $15(48,4)$ \\
\hline \multicolumn{3}{|c|}{ Acidente de trabalho } \\
\hline Sim & $2(4,4)$ & $2(6,5)$ \\
\hline Não & $43(95,6)$ & $29(93,5)$ \\
\hline \multicolumn{3}{|c|}{ Afastados por problemas de saúde } \\
\hline Sim & $5(11,1)$ & $3(9,7)$ \\
\hline Não & $40(88,9)$ & $28(90,3)$ \\
\hline \multicolumn{3}{|c|}{ Satisfação com o salário } \\
\hline Sim & $15(33,3)$ & $12(38,7)$ \\
\hline Não & $30(66,7)$ & $19(61,3)$ \\
\hline
\end{tabular}

Fonte: Banco de dados dos autores (2015).

Em relação a categoria de enfermeiros, $100 \%(\mathrm{n}=45)$ eram do sexo feminino, com idade média de 40,3 (DP=10,06), com variação de 27 a 67 anos. Quanto aos aspectos laborais, apresentaram mediana de 13 anos de formação, quatro anos de serviço e trabalhavam nos turnos manhã e tarde $(93,3 \%$; n=42). Apresentaram grau de satisfação de 75\% no trabalho $(55,6 \%$; $\mathrm{n}=25$ ), porém estavam insatisfeitos com o salário.

A categoria médica, prevaleceu sexo masculino $(67,7 \% ; n=21)$, com idade média de 43,71 ( $\mathrm{DP}=15,14)$, variando de 26 a 78 anos. Quanto aos aspectos laborais, apresentaram mediana de 17 anos de formação e dois anos de serviço, tinham outro emprego $(51,6 \%$; n=16) e trabalhavam nos turnos manhã e tarde $(67,7 \%$; $=21)$. Apresentaram grau de satisfação de $75 \%$ no trabalho $(45,2 \% ; n=14)$, porém estavam insatisfeitos com o salário.

Na Tabela 2, apresenta-se o valor do alfa de Cronbach das escalas EACT e EIPST e seus respectivos fatores e medidas de tendência central. 
Tabela 2. Média, desvio padrão e alfa de Cronbach para as escalas EACT e EIPST e seus respectivos fatores ( $\mathrm{n}=76$ ).

\begin{tabular}{llll} 
Escalas e Fatores & $\begin{array}{l}\text { Alfa de } \\
\text { Cronbach }\end{array}$ & Média \pm DP & \\
\cline { 3 - 4 } & & & \\
\cline { 3 - 4 } & & & \\
\hline EACT* & 0,92 & $3,45 \pm 0,47$ & $3,09 \pm 0,74$ \\
\hline Organização do Trabalho & 0,76 & $2,63 \pm 0,70$ & $2,24 \pm 0,70$ \\
Relações Socioprofissionais & 0,85 & $3,13 \pm 0,94$ & $2,87 \pm 1,03$ \\
Condições de Trabalho & 0,93 & & \\
\hline EIPST** & 0,89 & $3,81 \pm 1,24$ & $3,88 \pm 1,38$ \\
\hline Realização Profissional & 0,92 & $4,27 \pm 1,36$ & $4,01 \pm 1,22$ \\
Liberdade de Expressão & 0,87 & $3,60 \pm 1,42$ & $3,15 \pm 1,78$ \\
Esgotamento Profissional & 0,90 & $2,57 \pm 1,68$ & $2,0 \pm 1,82$ \\
Falta de Reconhecimento & 0,93 & &
\end{tabular}

*Escala de Avaliação do Contexto de Trabalho; **Escala de Indicadores de Prazer e Sofrimento no Trabalho. Fonte: Banco de dados dos autores (2015).

Na EACT, o alfa de cronbach variou de 0,76 a 0,93, com o valor de 0,92 para escala geral. Os enfermeiros e os médicos avaliaram o Contexto de Trabalho como moderado, crítico, exceto para o fator "Relações Socioprofissionais", avaliado somente pelos médicos como positivo e satisfatório.

Na EIPST, o alfa de cronbach variou de 0,87 a 0,93, com valor de 0,89 para escala geral. Conforme a avaliação dos enfermeiros e médicos, dois fatores da EIPST foram considerados moderado e crítico, tais quais: "Realização profissional" e "Esgotamento profissional". Somente o fator "Liberdade de expressão" foi considerado satisfatório para ambas as categorias. O fator "Falta de reconhecimento" foi satisfatório somente na avaliação dos médicos.

Na Tabela 3, apresenta-se as correlações entre os fatores das duas escalas, para as duas categorias em conjunto.

Tabela 3. Coeficiente de correlação entre os fatores da EACT e EIPST (n=76).

\begin{tabular}{llll}
\hline & \multicolumn{2}{l}{ Fatores de EACT } & \\
\cline { 2 - 4 } Fatores de EIPST & Organização do Trabalho & $\begin{array}{l}\text { Relações } \\
\text { Socioprofissionais }\end{array}$ & Condições de Trabalho \\
\hline Liberdade de expressão &,- 009 &,- 211 &,- 078 \\
Realização profissional &,- 218 &,$- 303 *$ &,- 196 \\
Esgotamento profissional & $\mathbf{, 5 1 2 *}$ &, $382^{*}$ &, $375^{*}$ \\
Falta de reconhecimento &, $419 *$ & $\mathbf{, 5 0 9 *}$ &, $362^{*}$ \\
\hline
\end{tabular}

*correlação significativa no nível 0,01. Fonte: Banco de dados dos autores (2015).

Observou-se correlação forte e significativa entre o fator "Organização do Trabalho" e "Esgotamento profissional" e entre "Relações socioprofissionais" com "Falta de reconhecimento". 


\section{Discussão}

A partir do perfil dos participantes, pode-se perceber que todos os enfermeiros eram do sexo feminino (100\%) com idade média de 40,3 anos, contudo na categoria médica, prevaleceram profissionais do sexo masculino, com idade média de 43,71 anos. A predominância do sexo feminino entre os profissionais de saúde é um dado muito frequente nas pesquisas, em especial na enfermagem, no entanto na área médica ainda é menos prevalente (Tambasco, Silva, Pinheiro \& Gutierrez, 2017; Arroyo-Laguna, 2020).

Quanto aos aspectos laborais, pode-se perceber que os profissionais de ambas categorias apresentaram maior tempo de experiência, ao levar em consideração a idade média dos participantes, o nível de ensino e o tempo de formação acadêmica. São profissionais que estão satisfeitos com o trabalho, a isso podem ser atribuídas ao aprimoramento da organização laboral nos últimos anos, no que se refere ao apoio da gestão e colegas em realizar as atividades nos setores (Suliman \& Aljezawi, 2018; Passadouro \& Ferreira, 2016). No entanto, são profissionais insatisfeitos com o salário, isso pode interferir na atuação dupla jornada desses trabalhadores (Tambasco, Silva, Pinheiro \& Gutierrez, 2017; Sha, Li, Law \& Yip, 2019) em especial, da categoria médica em que a maioria atua em mais de um emprego (51,6\%).

Para avaliar o contexto laboral, prazer e sofrimento nos trabalhadores de saúde da APS, utilizou-se as escalas EACT e EIPST. Para isso, foi necessário verificar a sua validade, a partir do cálculo da consistência interna de cada uma, em que apresentaram valores significativos e satisfatórios acima de 0,70 (Hair, Black, Babin, Anderson \&Tatham, 2009).

Outras pesquisas que utilizaram essas duas escalas apresentaram valores de consistência interna semelhantes, com variação de 0,68 a 0,91 para EACT (Lacerda, Ferreira, Bracarense, Sene \& Simões, 2016) e de 0,86 a 0,93 para EIPST, ambos estudos foram realizados em APS (Glanzner, Olschowsky, Pai, Tavares \& Hoffman, 2017). Dessa maneira, as escalas se mostraram válidas e fidedignas em avaliar o contexto de trabalho e a vivência de prazer e sofrimento entre enfermeiros e médicos da APS.

No que se refere à avaliação do contexto de trabalho, os enfermeiros e médicos da APS avaliaram como crítico, com risco moderado para o adoecimento, os fatores "Organização do trabalho" e "Condições de trabalho". Em concordância, estudo realizado em APS com a equipe de enfermagem, também identificou esses dois fatores como críticos, respectivamente $(\mathrm{M}=3,37 ; \mathrm{DP}=0,56)$ e ( $\mathrm{M}=2,75 ; \mathrm{DP}=0,82)$ (Lacerda, Ferreira, Bracarense, Sene \& Simões, 2016).

Esses resultados podem ser justificados pelo modo operacional dos serviços, em que a divisão de tarefas e ritmo de trabalho são, na maioria das vezes, mal distribuídas, devido à falta de profissionais capacitados que atuem nessas unidades (Sousa, Gonçalves, Silva, Soares, Nogueira \& Zeitoune, 2018; Mendes, Ferreira \& Cruz, 2007; Lacerda, Ferreira, Bracarense, Sene \& Simões, 2016).

No que diz respeito ao fator "Relações socioprofissionais", para categoria médica foi avaliado como satisfatório, contudo crítico para os enfermeiros. A diferença de avaliação entre essas categorias, para este fator, pode estar relacionada à pequena autonomia dos enfermeiros em exercer tarefas, tomar decisões diante de sua equipe e, de se comunicar com seus colegas e gestores de serviço (Lacerda, Ferreira, Bracarense, Sene \& Simões, 2016).

No que diz respeito a avaliação de indicadores de prazer e sofrimento no trabalho, evidenciou-se que os fatores "Realização profissional" e "Esgotamento profissional" foram considerados críticos pelos enfermeiros e médicos da APS. De acordo com estudo realizado no Sul do Brasil, com profissionais de saúde e de apoio, encontraram valores semelhantes, no entanto superiores a este estudo, sendo 3,85 $(\mathrm{DP}=137)$ para realização profissional e 3,56(DP=1,63) para o esgotamento profissional (Dalmolin, et al., 2020).

Os achados indicam que a criticidade desses fatores pode ser efeito do acúmulo de atividades e normatizações, a partir das exigências da instituição, que dificultam a refletir sobre a prática clínica (Glanzner, Olschowsky, Pai, Tavares \& Hoffman, 2017). A falta de realização e intenso esgotamento profissional, na maioria das vezes, é decorrente da ausência de valorização 
e reconhecimento, escassez de recursos humanos e de materiais, em que dificultam o fornecimento de uma assistência mais qualificada, o que torna o trabalho mais crítico (Dalmolin, et al., 2020; Glanzner, Olschowsky, Pai, Tavares \& Hoffman, 2017; Kolhs, Olschowsky, Barreta, Schimerfening, Vargas \& Busnello, 2017).

Quanto ao fator "Liberdade de expressão", único avaliado como satisfatório pelas duas categorias, pode-se inferir que esses trabalhadores atuam em local aberto para questionamentos, reflexão e organização de suas ideias além de, discussão sobre a prática clínica entre colegas e gestão (Pimenta, et al., 2020). Isso é indicativo de prazer para os trabalhadores, em que ao conseguirem se expressar e interagir com a equipe, com base na confiança reflete no cuidado junto aos usuários (Lamb, Beck, Coelho \& Vasconcelos, 2019; Machado, et al., 2015).

As vivências de injustiça, indignação, e desvalorização pelo não reconhecimento do trabalho do profissional compõem o fator "Falta de Reconhecimento", avaliado como crítico pelos enfermeiros e satisfatório pelos médicos. A diferença de avaliação entre os participantes deste estudo, pode estar relacionada à forma como cada categoria é tratada dentro desses setores, ao levar em consideração o prestígio da categoria médica, como centro do modelo assistencial biomédico ainda vigente (Glanzner, Olschowsky, Pai, Tavares \& Hoffman, 2017; Progianti, Souza, Oliveira, Rodrigues, Prata \& Vargens, 2019).

No que concerne a análise de correlações, observou-se forte correlação entre os fatores "Organização do trabalho" e "Esgotamento profissional". Tais resultados demonstram que melhorar a organização laboral parece ser um passo efetivo para reduzir o esgotamento profissional. A forma de organização do trabalho, com alta demanda para poucos profissionais em atuação (Miorin, Camponogara, Pinno, Beck, Costa \& Freitas, 2018), dificulta o fornecimento de um atendimento qualificado aos usuários. Essa realidade pode causar sofrimento no trabalhador, com base no esgotamento físico e psíquico (Tambasco, Silva, Pinheiro \& Gutierrez, 2017; Marques, Alves, Queirós, Norton \& Henriques, 2018).

Os fatores "Relações socioprofissionais" e "Falta de reconhecimento" também foram fortemente correlacionados entre si. A partir desse resultado, percebe-se que quando existe uma interação saudável entre colegas e gestão, com o reconhecimento de todos os profissionais, a partir de sua inclusão na resolução de problemas sobre o cuidado ao usuário, o ambiente de trabalho se torna mais leve e com menos risco de adoecimento (Lacerda, Ferreira, Bracarense, Sene \& Simões, 2016; Marques, Alves, Queirós, Norton \& Henriques, 2018).

\section{Conclusão}

No que se refere à avaliação do contexto de trabalho, os enfermeiros e médicos da APS avaliaram como crítico os fatores "Organização do trabalho" e "Condições de trabalho". O fator "Relações socioprofissionais", foi avaliado como satisfatório somente pela equipe médica.

Conforme os indicadores de prazer e sofrimento, os fatores "Realização profissional" e "Esgotamento profissional" foram avaliados como críticos. Somente o fator "Liberdade de expressão" foi considerado satisfatório, para ambas categorias. O fator "Falta de reconhecimento" foi satisfatório somente na avaliação dos médicos. No que diz respeito às correlações entre os fatores das duas escalas, observou-se correlação forte e significativa entre o fator "Organização do Trabalho" e "Esgotamento profissional" e entre "Relações socioprofissionais" e "Falta de reconhecimento".

Estas avaliações evidenciaram risco moderado de adoecimento no contexto de trabalho, em que enfermeiros sinalizaram, com avaliações mais críticas que os médicos, para os fatores de cada uma das escalas, exceto para o fator "Liberdade de expressão". Os resultados desta pesquisa vêm contribuir para o conhecimento acerca da saúde dos profissionais médicos e enfermeiros da APS e, poderá auxiliar gestores e trabalhadores na tomada de decisões que venham a promover melhorias nas múltiplas dimensões que envolvem o trabalho neste nível. 
A partir dos resultados deste estudo, sugere-se para pesquisas futuras desenvolvimento de ações de melhorias para proporcionar um ambiente de trabalho mais adequado, e com menor risco de adoecimento e sofrimento entre os profissionais da APS.

\section{Referências}

Arroyo-Laguna, J. (2020). Redistribution of salary or professional recognition? The difficult construction of a profession, the Peru vian nursing. Ciência \& Saúde Coletiva, 25(1), 223-232. http://dx.doi.org/10.1590/1413-81232020251.25972019

Bisquerra, R., Sarriera, J.C., \& Martínez, F (2004). Introdução à estatística: enfoque informático com o pacote estatístico SPSS. Artmed.

Brasil, Ministério da Saúde. (2017). Portaria n 2.436, de 21 de setembro de 2017. Diário Oficial da União.

Brasil, Ministério da Saúde. (2013). Conselho nacional de saúde, Comitê Nacional de Ética em Pesquisa em Seres Humanos. Resolução nº 466 de 12 de dezembro de 2012: Diretrizes e normas regulamentadoras para pesquisas envolvendo seres humanos. Brasília: Ministério da Saúde.

Dalmolin, G. L., et al (2020). Prazer e sofrimento em trabalhadores da atenção primária à saúde do Brasil. Rev Cuid, 11(1), e851. http://dx.doi.org/10.15649/cuidarte.851.

Dejours, C. (2004) Addendum: da psicopatologia à psicodinâmica do trabalho. In: Lancman S, Sznelwar LI. Christophe Dejours: da psicopatologia à psicodinâmica do trabalho. Brasília: Paralelo 15.

Dejours, C. A. (2013). Sublimação, entre sofrimento e prazer no trabalho. Revista Portuguesa de Psicanálise, 33(2), 9-28. https://pt.scribd.com/document/243253326/SUBLIMACAO-ENTRE-SOFRIMENTO-E-PRAZER-NO-TRABALHO-pdf.

Glanzner, C. H., Olschowsky, A., Pai, D. D, Tavares, J. P. \& Hoffman, D. A. (2017). Assessment of indicators and experiences of pain and pleasure in family health teams based on the Psychodynamics of Work. Rev Gaúcha Enferm, 38(4), e2017-0098. http://dx.doi.org/10.1590/1983-1447.2017.04.2017-0098.

Hair, J.F., Black, W.C., Babin, B.J., Anderson, R.E. \& Tatham, R.L. (2009). Análise multivariada de dados. Bookman.

Kolhs, M., Olschowsky, A., Barreta, N. L., Schimerfening, J., Vargas, R. \& Busnello, G.F. (2017). Nursing in urgency and emergency: between pleasure and suffering. Rev Fund Care Online, 9(2), 422-431. http://dx.doi.org/10.9789/2175-5361.2017.v9i2.422-431

Lacerda, R. B., Ferreira, M. B. G., Bracarense, C. F., Sene, L. V. \& Simões, A. L. A. (2016). Contexto de trabalho e Síndrome de Burnout na equipe de enfermagem da Estratégia Saúde da Família. Cultura de los Cuidados (Edición digital), 20, 44. http://dx.doi.org/10.14198/cuid.2016.44.08.

Lamb, F. A., Beck, C. L. C., Coelho, A. P. F. \& Vasconcelos, R. O. (2019). Nursing work in a pediatric emergency service: between pleasure and pain. Cogitare enferm, 24, e59396. http://dx.doi.org/10.5380/ce.v24i0.59396.

Machado, M. H. et al. (2015). Condições de trabalho da enfermagem. Enferm. Foco, 6 (1/4), 79-90. https://doi.org/10.21675/2357-707X.2016.v7.nESP.695

Marques, M. M., Alves, E., Queirós, C., Norton, P. \& Henriques, A. (2018). The effect of profession on burnout in hospital staff. Occupational Medicine, 68(3), 207-210. http://dx.doi.org/10.1093/occmed/kqy039.

Mendes, A. M., Ferreira, M. C. \& Cruz, R. M. (2007). Inventário sobre Trabalho e Riscos de Adoecimento-ITRA: Instrumento auxiliar de diagnóstico de indicadores críticos no trabalho. In: Mendes AM. Psicodinâmica do trabalho: teoria, método e pesquisas. São Paulo: Casa do Psicólogo, 1, 111-26.

Miorin, J, D, Camponogara, S., Pinno, C., Beck, C. L. C., Costa, V. \& Freitas, E. O. (2018). Pleasure and pain of nursing workers at a first aid service. Florianópolis (SC): Texto Contexto Enferm, 27(2), e2350015. http://dx.doi.org/10.1590/0104-070720180002350015.

Miranda, N. M. V., Rewa, T., Leonello, V. M., \& Oliveira, M.A.C. (2018). Advanced practice nursing: a possibility for Primary Health Care? Rev Bras Enferm, 71(Supl 1), 716-21. http://dx.doi.org/10.1590/0034-7167-2017-0672.

Oliveira, A. L. C. B., Costa, G. R., Fernandes, M. A, Gouveia, M. T. O. \& Rocha, S.S. (2018) Presenteeism: factors of risk and repercussions on the health of nursing workers. Av Enferm, 36(1), 79-87. http://dx.doi.org/10.1590/1518-8345.2458.3032

Passadouro, R. \& Ferreira, P. L. (2016). Professional Satisfaction within Family Health Units of Central Region. Acta Med Port, 29(11), 716-25. http://dx.doi.org/10.20344/amp.7380

Pereira, A. S., et al. (2018). Metodologia da pesquisa científica.

Pimenta, C. J. L. et al (2020). Pleasure and suffering among hospital nurses. Rev Bras Enferm, 73(2), e20180820. http://dx.doi.org/10.1590/0034-7167-20180820 .

Progianti, J. M., Souza, M. N., Oliveira, E. B., Rodrigues, F. A. B., Prata, J. A. \& Vargens, O. M. C. (2019). Suffering and pleasure experiences of nursing undergraduate students in maternity. Rev enferm UERJ, 27, e39620. http://dx.doi.org/10.12957/reuerj.2019.39620.

Sha, F., Li, B., Law, Y. W. \& Yip, P. S. F. (2019). Beyond the Resource Drain Theory: Salary satisfaction as a mediator between commuting time and subjective well-being. Journal of Transport \& Health, 15, 100631. https://doi.org/10.1016/j.jth.2019.100631

Schneider, A., Wehler, M. \& Weigl, M. (2019). Effects of work conditions on provider mental well-being and quality of care: a mixed-methods intervention study in the emergency department. BMC Emergency Medicine, 19(1). https://doi.org/10.1186/s12873-018-0218-x 
Research, Society and Development, v. 10, n. 2, e9610212331, 2021

(CC BY 4.0) | ISSN 2525-3409 | DOI: http://dx.doi.org/10.33448/rsd-v10i2.12331

Sousa, K. H. J. F., Gonçalves, T. S., Silva, M. B., Soares, E. C. F., Nogueira, M. L. F. \& Zeitoune, R. C. G. (2018). Risks of illness in the work of the nursing team in a psychiatric hospital. Rev. Latino-Am. Enfermagem, 26, e3032. http://dx.doi.org/10.1590/1518-8345.2458.3032.

Suliman, M. \& Aljezawi, M. (2018). Nurses' work environment: indicators of satisfaction. $J$ Nurs Manag, 26 (5), 525-530. http://dx.doi.org/10.1111/jonm.1257.

Tambasco, L. D., Silva, H. S., Pinheiro, K. M. \& Gutierrez, B. A. (2017). Satisfaction in the work of the multidisciplinary team which operates in Primary Health Care. Saúde em Debate, 41(n. especial), 140-151. http://dx.doi.org/10.1590/0103-11042017S212

Wagner, A., et al (2019). Healthcare professionals' perspectives on working conditions, leadership, and safety climate: a cross-sectional study. BMC Health Services Research, 19(53). https://doi.org/10.1186/s12913-018-3862-7. 